\title{
PEDIATRIC ATOPIC DERMATITIS: UPDATE ON THE TREATMENT OPTIONS
}

\author{
CORINA ADELINA ZAH ${ }^{1}$, ANDREEA EMILIANA TOPORĂ ${ }^{2}$, PAUL GRAMA $^{3}$ \\ 1,2,3 “Iuliu Haţieganu” University of Medicine and Pharmacy, Cluj-Napoca
}

\begin{abstract}
Keywords: pediatric Abstract: Atopic dermatitis (AD) is a chronic inflammatory disease characterized by skin dryness, atopic dermatitis, pruritus and eczematous lesions with various periods of relapse. Symptomatology can appear in pediatric eczema, childhood and can persist in adulthood. Chronic treatment is required with corticosteroids being the treatment updates, atopic standard options. The side effects of this type of long-term treatment represent a major concern for dermatitis treatment the pediatric patients. This review aims to give an update of the options used for treatment, apart from the systemic corticosteroids. Mild-to-moderate AD had a good response to creams containing fig and oatmeal extracts and inhibitors of phosphodiesterase-4 (crisaborole). In cases of severe AD, future treatment options could include monoclonal antibodies such as omalizumab and dupilumab
\end{abstract}

\section{INTRODUCTION}

Atopic dermatitis (AD), or atopic eczema, is a chronic inflammatory condition of the skin, with variable periods of relapse.(1,2) First manifestations can occur during childhood, and in most cases can persist into adulthood.(1,3) Prevalence has increased in the past years, in developed countries reaching 10$20 \%$.(1) Hallmarks of AD are represented by skin dryness, intense itching and erythematous, lichenified patches and plaques.(1,2) In children, eczematous lesions usually appear a few weeks after birth in characteristic locations: face (particularly the central cheeks), scalp, hands and feet, and extensor surfaces of the extremities. Xerosis, erythema, and papules, with or without serous exudate, is common, with itch being almost universal (particularly at night).(1,4) With age, lesions tend to become more dry and lichenified.(5) Serum IgE can be elevated, frequently there exists a personal or family history of atopy (asthma or allergic rhinitis).(6)

Pathogenesis of $\mathrm{AD}$ is multifactorial, involving genetic, immunological and environmental factors.(7) There is a decrease in the skin barrier function, associated with dehydration in the stratum corneum, impairment of its permeability and structural changes in lipids. $(3,8)$

Because of the chronic state of the disease, quality of life can be severely impaired manifesting with psychosocial stress, altered behaviour, sleep disturbances (alters motor and cognitive function) and infections. $(9,10)$

Severity of AD can be assessed using the SCORAD index which takes into account the severity of the lesions, pruritus and sleep disturbances.(7) Several sets of diagnostic criteria have been established, but the most used are the Hanifin \& Rajka and UK criteria.(11) Currently, treatment for $\mathrm{AD}$ is centred on avoiding irritants and allergens and using corticosteroids, but there is a trend towards other alternatives. This is mainly due to patient's and parent's interest in complementary and alternative medicine.

Treatment options vary according to disease severity. Pharmacological options include topic corticosteroids in mild and moderate cases. In severe AD the following can be administered: systemic corticosteroids, cyclosporine, azathioprine and methotrexate. It is necessary to keep in mind that certain triggers might be involved in reactivations and their elimination is important. During exacerbations of mild and moderate forms, treatment with topical corticosteroids (TCS) is recommended and after stabilization emollients should be used to prevent relapse. $(5,12)$

\section{AIM}

The aim of this review is to present different treatment options for pediatric $\mathrm{AD}$, apart from the standard corticosteroids.

\section{MATERIALS AND METHODS}

We performed a MEDLINE search for the following terms: "atopic dermatitis pediatric treatment", "atopic eczema", "atopic dermatitis", "atopic eczema alternative treatment", "childhood eczema treatment" using the following filters: Clinical Trial, Randomized Controlled Trial, published from January 1, 2015 to February 1, 2020 . A total of 130 articles were found, which were manually reviewed for inclusion criteria: articles written in English, addressing treatment on pediatric patients only. This resulted in 17 studies. Articles in which studies included also adults apart from children were not taken into account and studies trials that begun before 2013 were not cited as we desired to present the latest data related to subject.

\section{RESULTS}

Extracts from Ficus carica L. (fig) have been studied in form of a cream, in contrast to hydrocortisone $1 \%$ topic cream and placebo cream in children under 14 years old with mild to moderate AD. Figs are known to have effective constituents such as flavonoids, phenols, amino acids, zinc, calcium and magnesium. This is thought to give anti-inflammatory, antioxidant and antibacterial properties which may be effective in treating atopic dermatitis. Results showed a significant decrease in the SCORAD index after using the fig extract cream in

${ }^{1}$ Corresponding author: Corina Adelina Zah, Str. Louis Pasteur, Nr.4, Cluj Napoca, România, E-mail:corinazah@icloud.com, Phone: +40374 834114 Article received on 20.08.2020 and accepted for publication on 15.12.2020 


\section{CLINICAL ASPECTS}

comparison to the placebo cream and comparable effects with the hydrocortisone $1 \%$ cream. Such evidence should be further researched as it could potentially make for a good alternative treatment in pediatric patients with probably little to no side effects.(7)

Oatmeal benefits are largely studied as it poses possible anti-inflammatory properties. This is due to its capacity to inhibit nuclear factor kappa B in keratinocytes, which stimulates the release of pro-inflammatory cytokines and histamine, thus decreasing pruritus at a certain level. Apart from this, oat extracts contain avenanthramides, an anti-oxidant, which could be beneficial for AD. Several studies showed the efficacy of oatmeal extracts alone or in combination with other active ingredients. Over-the-counter $1 \%$ oatmeal cream improved symptomatology and hydration in children with mild-to-moderate AD. A cream containing colloidal oatmeal, avenanthramides, oat oil and shea butter had beneficial effects in moderate-to-severe AD. $(3,5)$ An alternative variant of cream, containing oatmeal in combination with zinc oxide, bisabolol and glycyrretinic acid was tested. Zinc oxide plays a role in restoring the skin barrier, bisabolol (an extract from German chamomile-Matricaria recutita) has antiinflammatory properties as well as glycyrretinic acid (obtained the glycyrrhizic acid from the herb liquorice). Results were found satisfying as the cream managed to reduce symptomatology in mild-to-moderate AD.(13)

Panthenol, a synthetic ingredient, acts by increasing ceramides, cholesterol and free fatty acids in stratum corneum, therefore restoring the skin barrier and promoting hydration. Use of topical emollient with panthenol for 3 months in children with mild $\mathrm{AD}$, in a stable phase, showed improvements of the objective and subjective SCORAD index.(8)

Some therapies target both $\mathrm{AD}$ and sleep disturbances: studies have been made on administration of melatonin. The efficacy of $6 \mathrm{mg}$ of melatonin given once a day for 6 weeks was tested in 2018. SCORAD index improved, but pruritus was not influenced by the melatonin administration. A similar study from 2016 used a 3mg dose of melatonin, once a day, but for 4 weeks. $(10,14)$

There is also the hypothesis that vitamin D plays a role in the pathogenesis of $\mathrm{AD}$, further increasing of the levels could be able lower the severity. Results are unclear regarding as some studies show paradoxal results. High levels of vitamin D are correlated with mild forms of $\mathrm{AD}$ while low levels correspond to severe AD. Intermediate concentrations may cause more severe lesions compared to lower levels.(2)

There are various studies centered on the efficacy of supplementation with Lactobacillus spp., some with contrasting results. Lactobacillus spp. are commonly found in the human gastrointestinal (GI) microbiota, skin and vagina. The amounts and types of species vary between the different structures of the GI tract. $(15,16)$ Probiotics are thought to play a role in increasing activity of regulatory $\mathrm{T}$ cells which may lead to suppressing inflammation. L.spp. also act by stimulating the mucin production in the epidermal layer, promoting activation of anti-inflammatory cytokines at a gastrointestinal level and stimulating production of IgA. As food allergies could trigger exacerbations of $\mathrm{AD}$, reducing gut inflammation is essential. Microbiota of children with $\mathrm{AD}$ is impaired as Clostridium spp. are represented in higher proportion, Bifidobacteria and L.spp. being less portrayed. This could also play a role in the inflammation, which could be balanced by supplementation of probiotics.(17,18,19,20)

Studies where L. rhamnosus and L. paracasei (strain GM-080), separately, were administered concomitant with TCS did not show any kind of improvements compared to topical medication alone, but these results could be influenced by the baseline TCS. $(4,18)$

A study from 2015 observed the effects of administering only Lactobacillus spp. to pediatric patients, in monotherapy. The trial included 4 groups: 1-L.paracasei, 2-L. fermentum, 3-mixture of first two, 4- placebo. Significant results showed a decrease in the SCORAD index in moderate-to-severe $\mathrm{AD}$ in all groups treated with L.spp. (17) Other mixture of probiotics which proved to be effective in treatment of moderate $\mathrm{AD}$ was made of $\mathrm{L}$. casei, Bifidobacterium lactis and $\mathrm{B}$. longum.(21)

Other targets involve phosphodiesterase-4, an intracellular enzyme which inhibited prevents activation of proinflammatory cytokines. Crisaborole (an inhibitor of phosphodiesterase-4) can be administered topically in form of an ointment of $2 \%$ concentration. This reduces adverse effects and facilitates compliance of the patient. Zane et al. showed the efficacy of topical application of crisaborole, signs and symptoms improving after 8 days of use. Few adverse effects were reported at the site of application, mainly healing spontaneously.(22)

Apart from TCS in forms of creams, wet-wrap dressings with ointment have been studied. In 2019, a trial compared the efficacy of TCS cream and wet-wrap dressings with $0.1 \%$ triamcinolone acetonide (TA). Dressings are characterized by first applying the ointment with TA and after applying a damp cotton cloth over the lesion for $30 \mathrm{~min}$. No differences have been noticed between the two methods, but patients related that they felt better with the dressings technique and they would prefer this kind of treatment in the future.(23)

A potential treatment option for severe AD could be use of Omalizumab (systemic administration- injection), an anti-IgE antibody. By binding to the IgE, it limits mast cell degranulation therefore decreasing release of inflammatory mediators. A randomized control trial compared placebo to Omalizumab. It is key to review that while the results were statistically significant, the change in objective SCORAD found was -6.9 (95\% CI, -12.2 to -1.5$)$ at 24 weeks, which is not clinically a large difference in disease severity compared to placebo. Another point is that patients with lower level of IgE did more favourably.(24) Further, IgE levels should be assessed before deciding on initiating therapy with this type of antibody.

Dupilumab, a monoclonal antibody that inhibits signalling of interleukin (IL)-4 and IL-13, was also studied on an adolescent population (younger than 18 years old). A decrease in the severity score was observed, compared to placebo.(25)

\section{DISCUSSIONS}

After consulting the current literature, an important number of alternative compounds were found as for treatment options for $\mathrm{AD}$, from mild to severe. In the case of pediatric patients, approaching chronic diseases is always a challenge from the compliance point of view, long term side effects and also parents' concerns. Choosing the best way of delivering an active substance with maximal benefits can difficult.

For mild-to-moderate $\mathrm{AD}$, natural alternatives such as creams with fig and oatmeal extracts could be of great value for pediatric patients, as adverse effects occurrence rates are in most cases close to zero if we exclude potential allergies.

Alternatives such as L.spp. supplementation or vitamin D supplementation are valuable for future references, especially in severe AD. Also, having other safe and efficient options apart from the classical immunosuppressive medication (corticosteroids, azthrioprine, methotrexate) might lead to a change in current guidelines.

\section{CONCLUSIONS}

Atopic dermatitis has become a very common affection of our days. Physicians should take into account the numerous alternatives to corticosteroids when prescribing a treatment to children to maximize compliance and efficacy. 


\section{CLINICAL ASPECTS}

REFERENCES

1. Weidinger S, Novak N. Atopic dermatitis. Lancet. 2016;387(10023):1109-22.

2. Lara-Corrales I, Huang CM, Parkin PC, Rubio-Gomez GA, Posso-De Los Rios CJ, Maguire J, et al. Vitamin D Level and Supplementation in Pediatric Atopic Dermatitis: A Randomized Controlled Trial. J Cutan Med Surg; 2018.

3. Diluvio L, Dattola A, Cannizzaro MV, Franceschini C, Bianchi L. Clinical and confocal evaluation of avenanthramides-based daily cleansing and emollient cream in pediatric population affected by atopic dermatitis and xerosis. $G$ Ital di Dermatologia e Venereol. 2019;154(1):32-6.

4. Yan DC, Hung CH, Sy LB, Lue KH, Shih IH, Yang CY, et al. A randomized, double-blind, placebo-controlled tria assessing the oral administration of a heat-treated lactobacillus paracasei supplement in infants with atopic dermatitis receiving topical corticosteroid therapy. Skin Pharmacol Physiol. 2019;32(4):201-11.

5. Lisante TA, Nuñez C, Zhang P. Efficacy and safety of an over-the-counter $1 \%$ colloidal oatmeal cream in the management of mild to moderate atopic dermatitis in children: a double-blind, randomized, active-controlled study. J Dermatolog Treat. 2017;28(7):659-67.

6. Eichenfield LF, Tom WL, Chamlin SL, Feldman SR, Hanifin JM, Simpson EL, et al. Guidelines of care for the management of atopic dermatitis: Section 1. Diagnosis and assessment of atopic dermatitis Work Group. J Am Acad Dermatol. 2014;70(2):338-51. .

7. Abbasi S, Kamalinejad M, Babaie D, Shams SM, Sadr Z Gheysari M, et al. A new topical treatment of atopic dermatitis in pediatric patients based on Ficus carica L. (Fig): A randomized, placebo-controlled clinical trial. Complement Ther Med. 2017;35(August):85-91

8. Stettler H, Kurka P, Kandzora J, Pavel V, Breuer M, Macura-Biegun A. A new topical panthenol-containing emollient for maintenance treatment of childhood atopic dermatitis: results from a multicenter prospective study. J Dermatolog Treat. 2017;28(8):774-9.

9. Wolter S, Price HN. Atopic Dermatitis. Pediatr Clin North Am [Internet]. 2014;61(2):241-60. Available from: http://dx.doi.org/10.1016/j.pcl.2013.11.002

10. Taghavi Ardakani A, Farrehi M, Sharif MR, Ostadmohammadi V, Mirhosseini N, Kheirkhah D, et al The effects of melatonin administration on disease severity and sleep quality in children with atopic dermatitis: A randomized, double-blinded, placebo-controlled trial. Pediatr Allergy Immunol. 2018;29(8):834-40.

11. Lee SC. Various diagnostic criteria for atopic dermatitis (AD): A proposal of Reliable Estimation of Atopic Dermatitis in Childhood (REACH) criteria, a novel questionnaire-based diagnostic tool for AD. J Dermatol. 2016;43(4):376-84.

12. Chan S, Cornelius V, Chen T, Radulovic S, Wan M, Jahan $\mathrm{R}$, et al. Atopic Dermatitis Anti-IgE Paediatric Trial (ADAPT): The role of anti-IgE in severe paediatric eczema: Study protocol for a randomised controlled trial. Trials. 2017;18(1):1-7.

13. Licari A, Ruffinazzi G, De Filippo M, CastaGnoli R, Marseglia A, Agostinis F, et al. A starch, glycyrretinic, zinc oxide and bisabolol based cream in the treatment of chronic mild-to-moderate atopic dermatitis in children: A threecenter, assessor blinded trial. Minerva Pediatr. 2017;69(6):470-5.

14. Chang Y Sen, Lin MH, Lee JH, Lee PL, Dai YS, Chu KH, et al. Melatonin Supplementation for Children with Atopic
Dermatitis and Sleep Disturbance: A Randomized Clinical Trial. JAMA Pediatr. 2016;170(1):35-42

15. Heeney DD, Gareau MG, Marco ML. Intestinal Lactobacillus in health and disease, a driver or just along for the ride? Curr Opin Biotechnol. 2018;49:140-7.

16. 1Zhang Z, Lv J, Pan L, Zhang Y. Roles and applications of probiotic Lactobacillus strains. Appl Microbiol Biotechnol. 2018;102(19):8135-43.

17. Wang IJ, Wang JY. Children with atopic dermatitis show clinical improvement after Lactobacillus exposure. Clin Exp Allergy. 2015;45(4):779-87.

18. Wu YJ, Wu WF, Hung CW, Ku MS, Liao PF, Sun HL, et al. Evaluation of efficacy and safety of Lactobacillus rhamnosus in children aged 4-48 months with atopic dermatitis: An 8-week, double-blind, randomized, placebocontrolled study. J Microbiol Immunol Infect. 2017;50(5):684-92

19. Kwon SH, Lim CJ, Jung J, Kim HJ, Park K, Shin JW, et al. The effect of autophagy-enhancing peptide in moisturizer on atopic dermatitis: a randomized controlled trial. J Dermatolog Treat. 2019;30(6):558-64

20. Mack DR, Ahrne S, Hyde L, Wei S, Hollingsworth MA. Extracellular MUC3 mucin secretion follows adherence of. Gut [Internet]. 2003;52:827-34.

21. Navarro-Lopez V, Ramirez-Bosca A, Ramon-Vidal D, Ruzafa-Costas B, Genoves-Martinez S, Chenoll-Cuadros E, et al. Effect of oral administration of a mixture of probiotic strains on SCORAD index and use of topical steroids in young patients with moderate atopic dermatitis a randomized clinical trial. JAMA Dermatology. 2018;154(1):37-43.

22. Zane LT, Kircik L, Call R, Tschen E, Draelos ZD, Chanda $\mathrm{S}$, et al. Crisaborole topical ointment, $2 \%$ in patients ages 2 to 17 years with atopic dermatitis: A phase $1 \mathrm{~b}$, open-label, maximal-use systemic exposure study. Pediatr Dermatol. 2016;33(4):380-7.

23. Cadmus SD, Sebastian KR, Warren D, Hovinga CA, Croce EA, Reveles LA, et al. Efficacy and patient opinion of wetwrap dressings using $0.1 \%$ triamcinolone acetonide ointment vs cream in the treatment of pediatric atopic dermatitis: A randomized split-body control study. Pediatr Dermatol. 2019;36(4):437-41.

24. Chan S, Cornelius V, Cro S, Harper JI, Lack G. Treatment Effect of Omalizumab on Severe Pediatric Atopic Dermatitis: The ADAPT Randomized Clinical Trial. JAMA Pediatr. 2020;174(1):29-37.

25. Simpson EL, Paller AS, Siegfried EC, Boguniewicz M, Sher L, Gooderham MJ, et al. Efficacy and Safety of Dupilumab in Adolescents with Uncontrolled Moderate to Severe Atopic Dermatitis: A Phase 3 Randomized Clinical Trial. JAMA Dermatology. 2020;156(1):44-56. 